\title{
Field Evaluation of Selected Insecticide Sequences against Two Cotton Bollworms with Reference to Side Effects on Coccinella undecimpunctata $\mathbf{L}$. Dalia A. El-Deeb ${ }^{1}$; Mervat H. A. Metayi ${ }^{2}$; Doaa A. Awad ${ }^{2}$ and A. F. Bedair ${ }^{1}$ \\ ${ }^{1}$ Central Pesticides Laboratory, Sabahia Station, Alexandria Egypt. \\ ${ }^{2}$ Plant Protection Research Institute, ARC, Sabahia, Alexandria, Egypt.
}

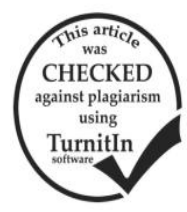

\section{ABSTRACT}

Field studies were conducted during 2015 and 2016 cotton growing seasons, at AbouElmatameer, El-Behira Governorate to evaluate certain insecticide sequences in controlling two cotton bollworms pink bollworm (PBW), Pectinophora gossypiella, (Saund) and spiny bollworm (SPW), Earias insulana, (Boisd.) The side effects of all insecticide sequences on the lady beetle, Coccinella undecimpunctata was also determined. During 2015 season, sequence 2 (Dursban ${ }^{\circledR}$, Rado- $\mathrm{X}^{\mathbb{B}}$, Radiant $^{\mathbb{B}}$, Cothrin ${ }^{\mathbb{B}}$ ) and sequence $6\left(\right.$ Dursban $^{\circledR}$, Rado- $^{\circledR}$, Radiant ${ }^{\circledR}$, Proclaim $\left.{ }^{\circledR}\right)$ achieved the highest efficacy against PBW, gave general mean reduction percentages 81.4 and $83.5 \%$, respectively. In season 2016, sequence 6 revealed the highest efficacy where the general mean reduction percentage was $83.3 \%$. Sequences 6 induced the highest reduction percentages in cotton bolls infested by SBW in 2015 and 2016 seasons, with general mean reduction percentages 84.0 and $82.3 \%$, respectively. Sequence 4 (Cothrin ${ }^{\circledR}$, Radiant $^{\mathbb{B}}$, Rado- $^{\circledR}$, Dursban $^{\circledR}$ ) achieved the least efficacy against PBW and SBW in both seasons 2015 and 2016. Sequence 6 proved to be the least toxic on C. undecimpunctata. On the other hand, all other sequences were comparable in there effects on $C$. undecimpunctata in both seasons.

\section{INTRODUCTION}

Cotton is grown primarily for fiber, but the seeds provide an important source of food for livestock and humans (Luttrell et al., 1994). In Egypt as well as in many countries, cotton liable to be attacked with different pests. Among these pests, are the most injurious insects: pink bollworm (PBW), Pectinophora gossypiella (Saund.), and spiny bollworm (SBW), Earias insulana (Boisd), (Ahmad et al., 2003; El-Aswad and Aly, 2007). When neglected, these two bollworms cause enormous damage and loss, qualitatively and quantitatively to the crop because they attacking cotton plants during flowering as well as fruiting stages (El-Feel et al., 1993). The production of cotton fibers depends mainly upon the efficient control of these insects. The control of these two insects relies mainly on the insecticide spraying. Pyrethroid, organophosphate and carbamate insecticide groups are commonly used for control of both PBW and SBW in cotton fields. But, the development of resistant strains by these insects against most or may be to all of these insecticide groups leads to the continuing need for new, effective and economical insecticides for crop protection. The phenylpyrazole insecticide fipronil and the spinosoid insecticide spinetoram are among the promising alternatives.

The fipronil mode of action differs from those of any other known agents. Fipronil has been reported to block both GABA receptors (Buckingham et al., 1994; Hosie et al., 1995) and insect inhibitory ionotropic glutamate receptors (Raymond et al., 2000). Spinetoram interacts with both $\gamma$-aminobutyric acid receptors and nicotinic acetylcholine receptors in a manner distinct from the interactions by other insecticides (Watson, 2001). It has recorded that fipronil and spinetoram achieved a good insecticidal activity against lepidopteran insects (Mulrooney, 2002; Kirst, 2010; Barrania et al., 2016). But, the success of cotton bollworm control programs relies mainly on the spraying insecticides belonging to different chemical families in a certain rotation. Also, the development of insecticide resistance may be reduced, by selecting products from different chemical families for an insecticide rotation program. So, the main purpose of this study was to incorporate fipronil in a suitable insecticide sequence, which gives a highest protection for cotton bolls against the infestation by the PBW and SBW. The side effects of these insecticide sequences on the lady beetle $C$. undecimpunctata were also determined.

\section{MATERIALS AND METHODS}

\section{Insecticides:}

Fipronil (Rado- $X^{\circledR} 80 \% \mathrm{WG}$ ), used at $40 \mathrm{gm} /$ fed., was produced by Jiangsu Tuoqiu Agrochemical Co. Spinetoram (Radiant ${ }^{\circledR} 12 \% \mathrm{SC}$ ), used at $100 \mathrm{ml} /$ fed., Spinosad (Tracer ${ }^{B} 24 \% \mathrm{SC}$ ), used at $100 \mathrm{ml} /$ fed., and chlorpyrifos (Dursban ${ }^{\circledR} 48 \%$ EC), used at 1 liter / fed., were produced by Dow Agrosciences Co. Deltamethrin (Cothrin ${ }^{\circledR} 10 \%$ EC), supplied by KZ company, was used at the rate of $500 \mathrm{ml} / \mathrm{fed}$. Emamectin benzoate (Proclaim $5 \% \mathrm{SG}$ ), used at $60 \mathrm{gm} / \mathrm{fed}$., was supplied by Syngenta.

\section{Field trials and the experimental design:}

Two field experiments were carried out during 2015 and 2016 summer seasons at AbouElmatameer, El-Behira Governorate. Cotton variety Giza 86 was cultivated at April 26, and April 30, during 2015 and 2016 seasons, respectively. All cultural practices were carried out according to "good agricultural practice". All treatments in addition to control were assigned to plots in a randomized complete block design with four replicates (each was $84 \mathrm{~m}^{2}$ in area). Plots have been separated from each by unplanted rows. Six insecticide sequences were arranged as presented in Table 1.

Table 1. Insecticide sequences used in 2015 and 2016 seasons

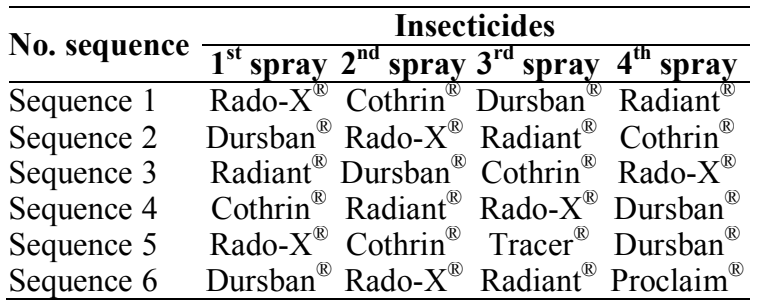

Insecticide applications were carried out using Knapsack sprayer equipment (CP3) at the rate of 200 liter per fed. Spraying took place at July 16 and August 1,15 \& 30, during 2015 cotton season and July 17 and August 1, 
$16 \& 30$, during cotton season 2016, respectively. Percentages of the two bollworm (PBW and SBW) infestations, each alone, were assessed according to the technique of El-Heneidy et al. (1987). Fifty green bolls were collected from each replicate (200 bolls from each treatment) at random from diagonals, where the counting was carried out before insecticides application and seven, and fourteen days after each spray. Boll samples were transferred to the laboratory, dissected and checked both externally and internally, and then percentages of boll infestations by PBW and that by SBW were calculated. At the same time, number of lady beetle was counted on ten cotton plants. The reduction percentages of PBW or SBW infestations which achieved by the treatments and the side effects on lady beetle were calculated according to Henderson and Tilton equation (1955). Data was presented as a mean for each insecticide spray and a general mean for each insecticides sequence. Means were compared for significance using analysis of variance (ANOVA) test (LSD at $\mathrm{P}<0.05)$ (SAS Statistical software, 1999).

\section{RESULTS}

Efficiency of the tested insecticide sequences against PBW:

Results shown in Tables ( 2 and 3 ) revealed that, all insecticide sequences achieved considerable reduction percentages of cotton bolls infested by PBW during the two seasons. During 2015 season, sequence 2 (Dursban ${ }^{\circledR}$, Rado- $X^{\circledR}$, Radiant ${ }^{\circledR}$, Cothrin $^{\circledR}$ ) and sequence 6 (Dursban ${ }^{\circledR}$, Rado- $X^{(}$, Radiant ${ }^{(}$, Proclaim $\left.{ }^{(}\right)$achieved the highest efficacy, gave the following reduction percentages (77.5, $85.8,83.5$ and $78.7 \%$ ) with general mean 81.4 and (78.5, $86.8,83.8,84.7)$ with general mean 83.5 , respectively. Sequence 4 (Cothrin $^{\circledR}$, Radiant ${ }^{\circledR}$, Rado- $X^{\circledR}$, Dursban ${ }^{\circledR}$ ) achieved the least efficacy, gave the following reduction percentages $(71.5,81.5,84.2,77.0)$ with general mean 78.6, respectively (Table 2). In season 2016, sequence 6 revealed the highest efficacy where the reduction percentages were $76.8,87.8,83.2$ and 85.4 with general mean 83.3 (Table 3).

Table 2. Efficacy of field application of different insecticide regimens on the cotton bolls infestation by $P$. gossypiella (season 2015)

\begin{tabular}{|c|c|c|c|c|c|}
\hline \multirow{2}{*}{ Treatments } & \multicolumn{5}{|c|}{ \% Reduction in the infestation of cotton bolls by $P$. gossypiella $\pm \mathrm{SE}$} \\
\hline & $1^{\text {st }}$ spray & $2^{\mathrm{IIU}}$ spray & $3^{\text {ru }}$ spray & $4^{\text {III }}$ spray & General mean \\
\hline Sequence 1 & $\begin{array}{c}\text { Rado-X } \\
83.1 \pm 2.5 \mathrm{a}\end{array}$ & $\begin{array}{c}\text { Cothrin } \\
76.4 \pm 3.7 \mathrm{~cd}\end{array}$ & $\begin{array}{c}\text { Dursban } \\
79.6 \pm 3.2 \mathrm{~b}\end{array}$ & $\begin{array}{l}\text { Radiant } \\
84.1 \pm 4.3 \mathrm{a}\end{array}$ & $80.8 \pm 3.7 \mathrm{bc}$ \\
\hline Sequence 2 & $\begin{array}{c}\text { Dursban } \\
77.5 \pm 1.9 \mathrm{~b}\end{array}$ & $\begin{array}{l}\text { Rado-X }^{\mathrm{w}} \\
85.8 \pm 2.4 \mathrm{a}\end{array}$ & $\begin{array}{c}\text { Radiant }^{(x)} \\
83.5 \pm 3.6 \mathrm{a}\end{array}$ & $\begin{array}{c}\text { Cothrin } \\
78.7 \pm 3.1 \mathrm{~b}\end{array}$ & $81.4 \pm 2.7 \mathrm{ab}$ \\
\hline Sequence 3 & $\begin{array}{c}\text { Radiant }^{(\mathbb{V})} \\
79.7 \pm 1.4 \mathrm{~b}\end{array}$ & $\begin{array}{l}\text { Dursban } \\
78.1 \pm 2.5 \mathrm{c}\end{array}$ & $\begin{array}{c}\text { Cothrin } \\
77.0 \pm 3.4 \mathrm{~b}\end{array}$ & $\begin{array}{l}\text { Rado-X } \\
84.3 \pm 2.9 \mathrm{a}\end{array}$ & $79.8 \pm 2.2 \mathrm{bc}$ \\
\hline Sequence 4 & $\begin{array}{l}\text { Cothrin } \\
71.5 \pm 3.6 \mathrm{c}\end{array}$ & $\begin{array}{l}\text { Radiant }^{(1)} \\
81.5 \pm 3.2 \mathrm{~b}\end{array}$ & $\begin{array}{c}\text { Rado-X }^{\text {(i) }} \\
84.2 \pm 3.4 \mathrm{a}\end{array}$ & $\begin{array}{l}\text { Dursban }^{(k)} \\
77.0 \pm 3.7 \mathrm{~b}\end{array}$ & $78.6 \pm 3.5 \mathrm{c}$ \\
\hline Sequence 5 & $\begin{array}{c}\text { Rado-X } X^{(\mathbb{k})} \\
82.9 \pm 3.9 \mathrm{a}\end{array}$ & $\begin{array}{c}\text { Cothrin } \\
75.0 \pm 2.8 \mathrm{~d}\end{array}$ & $\begin{array}{c}\text { Tracer }^{(k)} \\
78.2 \pm 2.7 \mathrm{~b}\end{array}$ & $\begin{array}{c}\text { Dursban } \\
76.8 \pm 3.6 \mathrm{~b}\end{array}$ & $78.2 \pm 2.8 \mathrm{c}$ \\
\hline Sequence 6 & $\begin{array}{c}\text { Dursban }^{\text {Du }} \\
78.5 \pm 3.2 \mathrm{~b}\end{array}$ & $\begin{array}{c}\text { Rado-X } \\
86.8 \pm 3.5 \mathrm{a}\end{array}$ & $\begin{array}{c}\text { Radiant }^{(\mathbb{}} \\
83.8 \pm 2.8 \mathrm{a}\end{array}$ & $\begin{array}{l}\text { Proclaim }^{(1)} \\
84.7 \pm 3.0 \mathrm{a}\end{array}$ & $83.5 \pm 3.4 \mathrm{a}$ \\
\hline
\end{tabular}

Efficiency of the tested insecticides sequences against SBW:

According to the statistical analysis, the exhibited data in Tables (3 and 4) demonstrated that sequences 6 induced the highest reduction percentages in cotton bolls infested by SBW in 2015 and 2016 seasons. Sequence 6 achieved reduction percentages $74.5,87.5,84.5$ and 89.5 with general mean 84.0 in 2015 and $71.5,85.7,83.4$ and 88.7 with general mean 82.3 in 2016 . In 2015 , sequence 4 recorded the least reduction percentages in cotton bolls infested by SBW in seasons 2015 with general mean $77.9 \%$. In 2016, sequence 2 and 4 recorded the least

Table 3. Efficacy of field application of different insecticide regimens on the cotton bolls infestation by $P$. gossypiella (season 2016)

\begin{tabular}{|c|c|c|c|c|c|}
\hline \multirow{2}{*}{ Treatments } & \multicolumn{5}{|c|}{$\%$ Reduction in the infestation of cotton bolls by $P$. gossypiella $\pm \mathrm{SE}$} \\
\hline & $1^{\text {st }}$ spray & $2^{\mathrm{InU}}$ spray & $3^{\text {ru }}$ spray & $4^{\text {tII }}$ spray & General mean \\
\hline Sequence 1 & $\begin{array}{c}\text { Rado-X } \\
82.6 \pm 3.7 \mathrm{a}\end{array}$ & $\begin{array}{l}\text { Cothrin } \\
76.5 \pm 1.9 \mathrm{~d}\end{array}$ & $\begin{array}{l}\text { Dursban } \\
78.4 \pm 2.5 \mathrm{bc}\end{array}$ & $\begin{array}{l}\text { Radiant } \\
81.2 \pm 2.1 \mathrm{bc}\end{array}$ & $79.7 \pm 2.8 b$ \\
\hline Sequence 2 & $\begin{array}{l}\text { Dursban } \\
75.2 \pm 3.7 \mathrm{c}\end{array}$ & $\begin{array}{c}\text { Rado-X } \\
86.4 \pm 4.1 \text { a }\end{array}$ & $\begin{array}{c}\text { Radiant }^{(k)} \\
82.5 \pm 2.9 \mathrm{a}\end{array}$ & $\begin{array}{c}\text { Cothrin } \\
77.2 \pm 3.2 \mathrm{~d}\end{array}$ & $80.3 \pm 3.1 \mathrm{~b}$ \\
\hline Sequence 3 & $\begin{array}{c}\text { Radiant }^{(1)} \\
79.5 \pm 1.8 \mathrm{~b}\end{array}$ & $\begin{array}{c}\text { Dursban } \\
78.1 \pm 3.8 \mathrm{~cd}\end{array}$ & $\begin{array}{l}\text { Cothrin } \\
76.0 \pm 2.8 \mathrm{c}\end{array}$ & $\begin{array}{c}\text { Rado-X } \\
84.5 \pm 2.7 \mathrm{ab}\end{array}$ & $79.5 \pm 3.1 \mathrm{~b}$ \\
\hline Sequence 4 & $\begin{array}{l}\text { Cothrin } \\
72.1 \pm 2.5 \mathrm{~d}\end{array}$ & $\begin{array}{l}\text { Radiant }^{(k)} \\
80.1 \pm 2.2 \text { bc }\end{array}$ & $\begin{array}{c}\text { Rado-X } \\
83.1 \pm 3.7 \mathrm{a}\end{array}$ & $\begin{array}{l}\text { Dursban } \\
78.3 \pm 2.6 \mathrm{~cd}\end{array}$ & $78.4 \pm 2.5 b$ \\
\hline Sequence 5 & 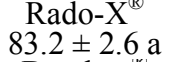 & $\begin{array}{c}\text { Cothrin }^{(k)} \\
77.2 \pm 2.5 \mathrm{~cd}\end{array}$ & $\begin{array}{c}\text { Tracer }^{(k)} \\
81.6 \pm 1.9 \mathrm{ab}\end{array}$ & $\begin{array}{l}\text { Dursban }{ }^{(1)} \\
77.9 \pm 2.8 \mathrm{~d}\end{array}$ & $80.0 \pm 3.1 \mathrm{~b}$ \\
\hline Sequence 6 & $\begin{array}{l}\left.\text { Dursban }^{(}\right) \\
76.8 \pm 2.1 \mathrm{c}\end{array}$ & $\begin{array}{c}\text { Rado-X } \\
87.8 \pm 3.8 \mathrm{a}\end{array}$ & $\begin{array}{l}\text { Radiant }^{(k)} \\
83.2 \pm 3.2 \mathrm{a}\end{array}$ & $\begin{array}{l}\text { Proclaim }^{(k)} \\
85.4 \pm 2.5 \mathrm{a}\end{array}$ & $83.3 \pm 2.7 \mathrm{a}$ \\
\hline
\end{tabular}

* Means within the same column followed by the same letters are not significantly different according to LSD at P $<0.05$. 
Table 4. Efficacy of field application of different insecticide regimens on the cotton bolls infestation by $E$. insulana (season 2015)

\begin{tabular}{|c|c|c|c|c|c|}
\hline \multirow{2}{*}{ Treatments } & \multicolumn{5}{|c|}{$\%$ Reduction in the infestation of cotton bolls by $E$. insulana $\pm \mathrm{SE}$} \\
\hline & $1^{\text {st }}$ spray & $2^{\text {IIU }}$ spray & $3^{\mathrm{ru}}$ spray & $4^{\text {III }}$ spray & General mean \\
\hline Sequence 1 & $\begin{array}{c}\text { Rado-X }^{\mathbb{N}} \\
88.3 \pm 3.7 \mathrm{a}\end{array}$ & $\begin{array}{c}\text { Cothrin } \\
77.4 \pm 1.5 \mathrm{c}\end{array}$ & $\begin{array}{l}\text { Dursban } \\
78.2 \pm 3.1 \mathrm{~b}\end{array}$ & $\begin{array}{l}\text { Radiant }^{\text {R }} \\
86.5 \pm 3.3 \mathrm{a}\end{array}$ & $82.6 \pm 2.7 \mathrm{a}$ \\
\hline Sequence 2 & $\begin{array}{l}\text { Dursban } \\
73.7 \pm 2.5 \mathrm{c}\end{array}$ & $\begin{array}{c}\text { Rado-X } \\
86.0 \pm 2.7 \mathrm{ab}\end{array}$ & $\begin{array}{l}\text { Radiant }^{(\pi)} \\
83.5 \pm 3.4 \mathrm{a}\end{array}$ & $\begin{array}{c}\text { Cothrin } \\
75.1 \pm 2.1 \mathrm{~b}\end{array}$ & $79.6 \pm 2.3 \mathrm{bc}$ \\
\hline Sequenc & $\begin{array}{l}\text { Radiant }^{(\sqrt{v})} \\
84.5 \pm 2.8 \mathrm{~b}\end{array}$ & $\begin{array}{l}\text { Dursban }^{\text {Dus }} \\
77.2 \pm 2.9 \mathrm{c}\end{array}$ & $\begin{array}{l}\text { Cothrin } \\
76.1 \pm 3.2 \mathrm{~b}\end{array}$ & $\begin{array}{c}\text { Rado-X }^{(\mathbb{k})} \\
88.4 \pm 3.4 \mathrm{a}\end{array}$ & $81.6 \pm 3.6 \mathrm{ab}$ \\
\hline Sequence 4 & $\begin{array}{c}\text { Cothrin } \\
69.3 \pm 2.5 \mathrm{~d}\end{array}$ & $\begin{array}{c}\text { Radiant }^{(\mathbb{a})} \\
83.0 \pm 3.2 \mathrm{~b}\end{array}$ & $\begin{array}{c}\text { Rado-X }{ }^{(1)} \\
84.1 \pm 3.5 \mathrm{a}\end{array}$ & $\begin{array}{c}\text { Dursban }^{\text {(a) }} \\
75.1 \pm 2.9 \mathrm{~b}\end{array}$ & $77.9 \pm 2.2 \mathrm{c}$ \\
\hline Sequence 5 & $\begin{array}{l}\text { Rado-X } \\
87.4 \pm 2.3 \mathrm{a}\end{array}$ & $\begin{array}{l}\text { Cothrin } \\
78.0 \pm 1.9 \mathrm{c}\end{array}$ & $\begin{array}{c}\text { Tracer }^{*} \\
85.6 \pm 2.4 \mathrm{a}\end{array}$ & $\begin{array}{l}\text { Dursban } \\
76.8 \pm 2.5 \mathrm{~b}\end{array}$ & $82.0 \pm 2.6 \mathrm{ab}$ \\
\hline Sequence 6 & $\begin{array}{c}\text { Dursban }^{*} \\
74.5 \pm 2.9 \mathrm{c}\end{array}$ & $\begin{array}{c}\text { Rado-X } \\
87.5 \pm 3.5 \mathrm{a}\end{array}$ & $\begin{array}{c}\operatorname{Radiant}^{(1)} \\
84.5 \pm 2.8 \mathrm{a}\end{array}$ & $\begin{array}{l}\text { Proclaim }^{(1)} \\
89.5 \pm 3.2 \mathrm{a}\end{array}$ & $84.0 \pm 3.1 \mathrm{a}$ \\
\hline
\end{tabular}

Table 5. Efficacy of field application of different insecticide regimens on the cotton bolls infestation by $E$. insulana (season 2016)

\begin{tabular}{|c|c|c|c|c|c|}
\hline \multirow{2}{*}{ Treatments } & \multicolumn{5}{|c|}{$\%$ Reduction in the infestation of cotton bolls by $E$. insulana $\pm \mathrm{SE}$} \\
\hline & $1^{s}$ spray & $2^{\ln }$ spray & $3^{\text {ru }}$ spray & $4^{\text {"II }}$ spray & General mean \\
\hline Sequence 1 & $\begin{array}{c}\text { Rado-X } \\
86.5 \pm 2.5 \mathrm{a}\end{array}$ & $\begin{array}{c}\text { Cothrin } \\
73.6 \pm 3.1 \mathrm{c}\end{array}$ & $\begin{array}{c}\text { Dursban } \\
74.1 \pm 2.8 \mathrm{~b}\end{array}$ & $\begin{array}{c}\text { Radiant } \\
85.0 \pm 3.0 \mathrm{~b}\end{array}$ & $79.8 \pm 3.1 \mathrm{bc}$ \\
\hline Sequence 2 & $\begin{array}{l}\text { Dursban } \\
70.4 \pm 1.8 \mathrm{c}\end{array}$ & $\begin{array}{l}\text { Rado-X } \\
84.6 \pm 3.2 \mathrm{a}\end{array}$ & $\begin{array}{l}\text { Radiant }^{(5)} \\
82.7 \pm 3.8 \mathrm{a}\end{array}$ & $\begin{array}{l}\text { Cothrin } \\
71.5 \pm 2.7 \mathrm{c}\end{array}$ & $77.3 \pm 3.5 \mathrm{c}$ \\
\hline Sequence 3 & $\begin{array}{l}\text { Radiant }^{(\sqrt{k})} \\
82.6 \pm 3.5 \mathrm{~b}\end{array}$ & $\begin{array}{c}\text { Dursban } \\
74.5 \pm 2.4 \mathrm{c}\end{array}$ & $\begin{array}{l}\text { Cothrin } \\
71.6 \pm 2.8 \mathrm{~b}\end{array}$ & $\begin{array}{c}\text { Rado-X } \\
84.6 \pm 3.9 \text { b }\end{array}$ & $78.3 \pm 2.5 \mathrm{bc}$ \\
\hline Sequence 4 & $\begin{array}{c}\text { Cothrin } \\
65.4 \pm 2.8 \mathrm{~d}\end{array}$ & $\begin{array}{l}\text { Radiant }^{(k)} \\
81.3 \pm 2.5 \mathrm{~b}\end{array}$ & $\begin{array}{c}\text { Rado-X } \\
85.7 \pm 2.9 \text { a }\end{array}$ & $\begin{array}{l}\text { Dursban } \\
72.5 \pm 2.4 \mathrm{c}\end{array}$ & $76.2 \pm 2.8 \mathrm{c}$ \\
\hline Sequence & $\begin{array}{l}\text { Rado-X } \\
86.9 \pm 3.5 \text { a }\end{array}$ & $\begin{array}{c}\text { Cothrin } \\
73.9 \pm 2.6 \mathrm{c}\end{array}$ & $\begin{array}{c}\text { Tracer }^{k} \\
84.9 \pm 2.2 \mathrm{a}\end{array}$ & $\begin{array}{l}\text { Dursban } \\
74.5 \pm 2.8 \mathrm{c}\end{array}$ & $80.1 \pm 2.4 \mathrm{ab}$ \\
\hline Sequence 6 & $\begin{array}{c}\text { Dursban } \\
71.5 \pm 2.3 \mathrm{c}\end{array}$ & $\begin{array}{c}\text { Rado-X } \\
85.7 \pm 3.1 \mathrm{a}\end{array}$ & $\begin{array}{l}\text { Radiant }^{(\pi)} \\
83.4 \pm 3.7 \mathrm{a}\end{array}$ & $\begin{array}{l}\text { Proclaim } \\
88.7 \pm 2.9 \mathrm{a}\end{array}$ & $82.3 \pm 2.7 \mathrm{a}$ \\
\hline
\end{tabular}

*Means within the same column followed by the same letters are not significantly different according to LSD at $P<0.05$.

Table 6. Side effects of different insecticide regimens on the $C$. undecimpunctata in cotton fields (season 2015)

\begin{tabular}{|c|c|c|c|c|c|}
\hline \multirow{2}{*}{ Treatments } & \multicolumn{5}{|c|}{ \% Reduction in the infestation of cotton bolls by $C$. undecimpunctata $\pm \mathrm{SE}$} \\
\hline & $1^{\text {st }}$ spray & $2^{110}$ spray & $3^{\text {ru }}$ spray & $4^{\mathrm{II}}$ spray & General mean \\
\hline Sequence 1 & $\begin{array}{c}\text { Rado-X } \\
18.5 \pm 1.1 \mathrm{c}\end{array}$ & $\begin{array}{c}\text { Cothrin } \\
31.4 \pm 1.5 \mathrm{bc}\end{array}$ & $\begin{array}{c}\text { Dursban } \\
26.3 \pm 2.1 \mathrm{c}\end{array}$ & $\begin{array}{c}\text { Radiant } \\
36.6 \pm 2.5 \mathrm{a}\end{array}$ & $28.2 \pm 2.7 \mathrm{a}$ \\
\hline Sequence 2 & $\begin{array}{l}\text { Dursban } \\
28.2 \pm 2.3 \mathrm{~b}\end{array}$ & $\begin{array}{c}\text { Rado-X } \\
17.4 \pm 1.7 \mathrm{~d}\end{array}$ & $\begin{array}{l}\text { Radiant }^{(\mathbb{k}} \\
37.5 \pm 2.1 \mathrm{a}\end{array}$ & $\begin{array}{l}\text { Cothrin } \\
33.8 \pm 2.7 \mathrm{~b}\end{array}$ & $29.2 \pm 2.2 \mathrm{a}$ \\
\hline Sequence 3 & $\begin{array}{l}\text { Radiant }^{(\mathbb{V}} \\
37.1 \pm 2.8 \mathrm{a}\end{array}$ & $\begin{array}{l}\text { Dursban }^{(1)} \\
29.4 \pm 2.6 \mathrm{c}\end{array}$ & $\begin{array}{c}\text { Cothrin } \\
34.0 \pm 1.7 \mathrm{~b}\end{array}$ & $\begin{array}{l}\text { Rado-X } \\
19.5 \pm 1.1 \mathrm{~d}\end{array}$ & $30.0 \pm 2.6 \mathrm{a}$ \\
\hline Sequence 4 & $\begin{array}{l}\text { Cothrin } \\
34.7 \pm 3.0 \mathrm{a}\end{array}$ & $\begin{array}{l}\text { Radiant }^{(k)} \\
42.6 \pm 2.8 \mathrm{a}\end{array}$ & $\begin{array}{c}\text { Rado-X } \\
18.5 \pm 1.0 \mathrm{~d}\end{array}$ & $\begin{array}{l}\text { Dursban }^{(k)} \\
31.1 \pm 2.5 \mathrm{~b}\end{array}$ & $31.7 \pm 2.1 \mathrm{a}$ \\
\hline Sequence 5 & $\begin{array}{c}\text { Rado-X } \\
17.4 \pm 1.6 \mathrm{c}\end{array}$ & $\begin{array}{c}\text { Cothrin } \\
34.2 \pm 1.2 \mathrm{~b}\end{array}$ & $\begin{array}{c}\text { Tracer } \\
37.5 \pm 2.7 \mathrm{a}\end{array}$ & $\begin{array}{l}\text { Dursban } \\
24.1 \pm 2.2 \mathrm{c}\end{array}$ & $28.3 \pm 2.6 \mathrm{a}$ \\
\hline Sequence 6 & $\begin{array}{c}\text { Dursban } \\
27.5 \pm 2.4 \mathrm{~b}\end{array}$ & $\begin{array}{l}\text { Rado-X } \\
17.9 \pm 1.2 \mathrm{~d}\end{array}$ & $\begin{array}{c}\text { Radiant }^{(1)} \\
37.2 \pm 2.9 \mathrm{a}\end{array}$ & $\begin{array}{l}\text { Proclaim } \\
10.2 \pm 1.0 \mathrm{e}\end{array}$ & $23.2 \pm 2.0 \mathrm{~b}$ \\
\hline
\end{tabular}

*Means within the same column followed by the same letters are not significantly different according to LSD at $P<0.05$.

Table 7. Side effects of different insecticide regimens on the $C$. undecimpunctata in cotton fields (season 2016)

\begin{tabular}{|c|c|c|c|c|c|}
\hline \multirow{2}{*}{ Treatments } & \multicolumn{3}{|c|}{$\%$ Reduction in the infestation of cotton bolls $t$} & \multicolumn{2}{|c|}{ C. undecimpunctata $\pm \mathrm{SE}$} \\
\hline & $1^{\text {st }}$ spray & $2^{\text {na }}$ spray & $3^{\mathrm{ru}}$ spray & $4^{\mathrm{III}}$ spray & General mean \\
\hline Sequence 1 & $\begin{array}{c}\text { Rado-X } \\
16.8 \pm 1.7 \mathrm{~d}\end{array}$ & $\begin{array}{c}\text { Cothrin } \\
33.1 \pm 3.2 \mathrm{c}\end{array}$ & $\begin{array}{c}\text { Dursban } \\
25.6 \pm 2.5 \mathrm{~b}\end{array}$ & $\begin{array}{c}\text { Radiant } \\
38.2 \pm 3.7 \mathrm{a}\end{array}$ & $28.4 \pm 1.9 \mathrm{a}$ \\
\hline Sequence 2 & $\begin{array}{c}\text { Dursban }^{(1)} \\
29.8 \pm 2.8 \mathrm{c}\end{array}$ & $\begin{array}{l}\text { Rado-X } \\
18.9 \pm 1.4 \mathrm{e}\end{array}$ & $\begin{array}{l}\text { Radiant } \\
41.8 \pm 3.6 \mathrm{a}\end{array}$ & $\begin{array}{c}\text { Cothrin } \\
35.2 \pm 2.4 \mathrm{a}\end{array}$ & $31.4 \pm 2.5 \mathrm{a}$ \\
\hline Sequence 3 & $\begin{array}{c}\text { Radiant } \mathrm{t}^{(\pi)} \\
39.5 \pm 3.2 \mathrm{a}\end{array}$ & $\begin{array}{c}\text { Dursbann }^{(1)} \\
28.4 \pm 2.3 \mathrm{~d}\end{array}$ & $\begin{array}{c}\text { Cothrin } \\
37.2 \pm 2.8 \mathrm{a}\end{array}$ & $\begin{array}{c}\text { Rado- } X^{*} \backslash \\
21.7 \pm 2.0 \mathrm{c}\end{array}$ & $31.7 \pm 2.2 \mathrm{a}$ \\
\hline Sequence 4 & $\begin{array}{c}\text { Cothrin }^{(1)} \\
33.5 \pm 2.3 \mathrm{~b}\end{array}$ & $\begin{array}{c}\text { Radiant }^{(1)} \\
41.9 \pm 3.4 \mathrm{a}\end{array}$ & $\begin{array}{c}\left.\text { Rado-X }{ }^{(}\right) \\
17.8 \pm 1.6 \mathrm{c}\end{array}$ & $\begin{array}{l}\left.\text { Dursban }^{(\mathbb{}}\right) \\
30.5 \pm 1.9 \mathrm{~b}\end{array}$ & $30.9 \pm 2.5 \mathrm{a}$ \\
\hline Sequence 5 & $\begin{array}{c}\text { Rado-X } \\
16.7 \pm 1.4 \mathrm{~d}\end{array}$ & $\begin{array}{c}\text { Cothrin } \\
37.9 \pm 2.8 \mathrm{~b}\end{array}$ & $\begin{array}{c}\text { Tracer }{ }^{(k)} \\
39.6 \pm 3.2 \mathrm{a}\end{array}$ & $\begin{array}{c}\text { Dursban }^{(k)} \\
27.8 \pm 2.7 \mathrm{~b}\end{array}$ & $30.5 \pm 2.8 \mathrm{a}$ \\
\hline Sequence 6 & $\begin{array}{c}\text { Dursban }^{(1)} \\
29.8 \pm 1.9 \mathrm{c}\end{array}$ & $\begin{array}{c}\left.\text { Rado-X }{ }^{(}\right) \\
15.8 \pm 1.6 \mathrm{e}\end{array}$ & $\begin{array}{c}\text { Radiant } \mathrm{R}^{(\mathbb{}} \\
38.5 \pm 2.9 \mathrm{a}\end{array}$ & $\begin{array}{l}\text { Proclaim }^{(1)} \\
11.5 \pm 1.2 \mathrm{~d}\end{array}$ & $23.9 \pm 2.7 b$ \\
\hline
\end{tabular}

*Means within the same column followed by the same letters are not significantly different according to LSD at $P<0.05$.

\section{DISCUSSION}

Since the cotton bollworms are almost present during the whole cycle of cotton, it is therefore relatively more expensive because repeated spraying is necessary. Moreover, resistance of key insect pests to insecticides continues to be a significant problem in cotton production
(Cook et al., 2005). For this reason, there is a greater need to develop alternative insecticides or additional techniques, which would allow a rational use of pesticides and provides adequate crop protection for sustainable food, feed and fiber production. Fipronil and spinetoram exhibited a good efficacy when it was evaluated each alone 
against PBW and SBW (Barrania et al., 2016). But, the success of cotton bollworm control programs relies mainly on the spraying insecticides belonging to different chemical families with different modes of action in a certain rotation. Therefore, this study was carried out to evaluate fipronil and spinetoram in different insecticide sequences to choose the most effective on in controlling PBW and SBW.

In the present study, the best results in controlling PBW and SBW were obtained when the insecticide sequence was started by the organophosphate insecticide Dursban ${ }^{\circledR}$ followed by Rado-X ${ }^{\circledR}$ which is followed by Radiant ${ }^{\circledR}$ which is followed by Proclaim $\AA$ (sequences 6). These results were similar to the results of Abou-Kahla et al. (1992), who reported that good results were achieved against the cotton bollworms by starting the insecticide sequence with the carbamate insecticide Larvin. Also, ElFeel et al. (1993) reported that thiodicarb and methomyl can provide a good tool to prevent the building-up of the PBW population at the early season. It is also recorded that, starting the insecticide program by the organophosphorus insecticide Dursban (chlorpyrifos) revealed a highly reduction percentages of the PBW and SBW numbers infesting the green bolls (El-Dessouki et al., 2006). In addition, this finding is in agreement with those of Tadros (2003) who showed that the starting of insecticide sequence with the organophosphorus compound chlorpyrifos gave high reduction of SBW infestation.

Using Proclaim ${ }^{\circledR}$ (emamectin benzoate) as an alternative to the pyrethroid insecticide Coathrin ${ }^{\mathbb{B}}$ (deltamethrin) improved the efficiency of the insecticide sequence against both insects PBW and SBW (sequence 6). Emamectin benzoate is novel semi-synthetic derivative of the natural product abamectin from the avermectin family of 16-membered macrocyclic lactones. This epi-methyl amino derivative is very effective against a broad spectrum of lepidopteran pests with good field efficacy and lack of cross-resistance with other commercially-used pesticides (White et al., 1997). The mode of action of emamectin benzoate is similar to abamectin (a GABA and glutamate-gated chloride channel agonist) according to Dunbar et al. (1998). Saleh et al., (2013) mentioned that, emamectin benzoate achieved high efficacy against PBW and SBW insects. Gupta et al., (2005) and Sontakke et al., (2007) reported that, emamectin benzoate was the most potent treatment in reducing pink bollworm, PBW and SBW.

The least results in controlling PBW and SBW were achieved when the insecticide sequence was started by the pyrethroid insecticide deltamethrin or spinosad (sequences 3 and 4). El-Gogary (1987) and El-Feel et al. (1990) mentioned that pyrethroids could not able to reduce the building-up of the PBW population at the early of season. Results of the present study were in partial agreement with El-Feel et al. (1993), who recorded that starting or mediating the insecticide sequence with pyrethroids depress the efficiency of that sequence.

Finally, to overcome or reduce the incidence of insecticide resistance, it is important to rotate between insecticides from different chemical families, particularly if several insecticide applications are made in a season.
Insecticides in different chemical families generally kill insects in different ways, whereas insecticides in the same chemical family often kill insects in the same way. Insects that survive application of a particular insecticide may be killed by an insecticide that kills the insect differently. By selecting products from different chemical families for an insecticide rotation program, the development of insecticide resistance may be reduced and increases the life of insecticides. In general this study pointed out to minimize repetition of insecticide application in the same season, furthermore using different insecticide sequences including fipronil, spinetoram \& emamectin benzoate and looking forward to an integrated pest management to overcome pest problems.

\section{REFERENCES}

Abou-Kahla, M. M., A. E. M. El-Sorady, R. M. Salem and A. M. Hussain (1992). Impact of several sequences of insecticides against certain cotton pests and the associated predators in cotton fields. J. Agric. Res. Tanta Univ. 18: 802-817.

Ahmad, M., Arif, M. I., Ahmad, Z., 2003. Susceptibility of Helicoverpa armigera (Lepidoptera: Noctuidae) to new chemistries in Pakistan. Crop Protection. 22, 539-544.

Barrania, A. A., M. H. A. Metayi and H. K. Abou-Taleb (2016). Field evaluation of some insecticide treatments against cotton bollworms and their side effects on two natural enemies. Egyptian Scientific J. Pesticides. 2: 27-36.

Buckingham, S. D., A. M. Hosie, R. L. Roush and D. B. Sattelle. 1994. Actions of agonists and convulsant antagonists on a Drosophila melanogaster GABA receptor (Rdl) homo-oligomer expressed in Xenopus oocytes. Neurosci Lett. 181: 137-40.

Cook, D. R., B. R. Leonard, J. Gore and J. H. Temple (2005). Baseline responses of bollworm, Helicoverpa zea (Boddie), and tobacco budworm, Heliothis virescens (F.), to indoxacarb and pyridalyl. J. Agric. Urban Entomol. 22: 99-109.

Dunbar, D. M., D. S. Lawson, S. M. White, N. Ngo, P. Dugger and D. Richter (1998). Emamectin benzoate: control of the heliothine complex and impact on beneficial arthropods, In: Proceedings of the 1998 Beltwide Cotton Conference, San Diego, California, USA. pp.1116-1118.

El-Aswad, A. F., Aly, M. I., 2007. Screening of some insecticides against the cotton bollworms, Pectinophora gossypilla (Saund.) and Earias insulana (Boisd.). J. Pest Cont. Environ. Sci. 15, 63-74.

El-Dessouki, S. A. I., A. S. El-Khouly, A. A. S. El-Zanan and H. M. H. Somaa (2006). Effect of some insecticides sequences against cotton bollworms larvae, non-target insects and associated predators in cotton fields. Egypt J. Agric. Res. 84: 1451-1462.

El-Feel, E. A., A. A. Khidr, M. M. Abou-Kahla and M. G. Abbass (1990). Effect of sequence, time intervals and early spray of different insecticides on the pink bollworm Pectinophora gossypiella (Saunders) infestation and the cotton yield. Agric. Res. Rev. Egypt. 
El-Feel, E. A., A. E. M. El-Sorady, H. A. Awad and M. E. Omar (1993). Influence of certain insecticide regimes against bollworms and other cotton pests and their predators. Alex. Sci. Exch. 14: 145-165.

El-Gogary, O. A. (1987). Effect of pheromones and chemical pesticides on population density of cotton bollworms. Ph. D. Thesis. Fac. Agric. Helwan Univ.

El-Heneidy, A. H., M. S. T. Abbas and A. A. Khidr. 1987. Comparitive population densities of certain predators in cotton fields treated with sex pheromones and insecticides in Minufya governorate. Egypt Bull. Entomol. Soc. 16: 181-190.

Gupta, G.P.; M. Raghuraman; A. B. and B. Singh (2005). Field efficacy of newer insecticides against bollworms in cotton. Indian. J. Entomol., 67: 16-20.

Henderson, C. F. and E. W. Telton. 1955. Tests with acaricides against the brown wheat mite. J. Econ. Entomol. 48, 157-161.

Hosie, A. M., H. A. Baylis, S. D. Buckingham and D. B. Sattelle. 1995. Actions of the insecticide fipronil, on dieldrin-sensitive and -resistant GABA receptors of Drosophila melanogaster. Br. J. Pharmacol. 115: 909-12.

Kirst, H. A. 2010. The spinosyn family of insecticides: realizing the potential of natural products research. The Journal of Antibiotics. 63: 101-111.

Luttrell, R. G., G. P. Fitt, F. S. Ramalho and E. S. Sugonyaev (1994). Cotton pest management: Part 1. A worldwide perspective. Annu. Rev. Entomol. 39: 517-526.

Mulrooney, J. E. 2002. Efficacy of fipronil aerially applied in oil adjuvants and drift retardants against boll weevils, Anthonomus grandis Boheman (Coleoptera: Curculionidae). Southwestern Entomologist. 27: 201207.
Raymond, V., D. B. Sattelle and B. Lapied (2000). Coexistence in DUM neurones of two $\mathrm{GluCl}$ channels that differ in their picrotoxin sensitivity. Neuro Report. 11: 2695-701.

Saleh, A. A.; L. R. Elgohary; W. M. Watson and A. S. Elabasy (2013). Efficiency of some new insecticides on cotton bollworms, Pectinophora gossypiella (Saund.) and Earias insulana(boisd.). J. Plant Prot. and Path., Mansoura Univ. 4: 617 624.

SAS Institute, Inc. (1999). PC — SAS users guide, Version 8. North Carolina statistical analysis system Institute, Inc.

Sontakke, B.K.; N. Das and L.K. Swain (2007). Bioefficacy of emamectin benzoate against bollworm complex in cotton. Ann. Pl. Protec. Sci., 15: 1 -3.

Tadros (2003). Effect of some insecticides on cotton bollworms and on quantity and quality of cotton yield. M. Sc. Thesis, Fac. Agric. (Saba-Bacha), Alex. Univ., Alexandria, Egypt

Watson, G. B. 2001. Actions of insecticidal spinosyns on $\gamma$ aminobutyric acid responses from small-diameter cockroach neurons. Pest. Biochem. Physiol. 71: 2028.

White, S. M., D. M. Dunbar, R. Brown, B. Cartwright, D. Cox, C. Eckel, R. K. Jansson, P. K. Mookerjee, J. A. Norton, R. F. Peterson and V. R. Starner (1997). Emamectin benzoate: a novel derivate for control of lepidopterous pests in cotton. In: Proceedings of Beltwide Cotton Conferences, New Orleans, pp.1078-1082.

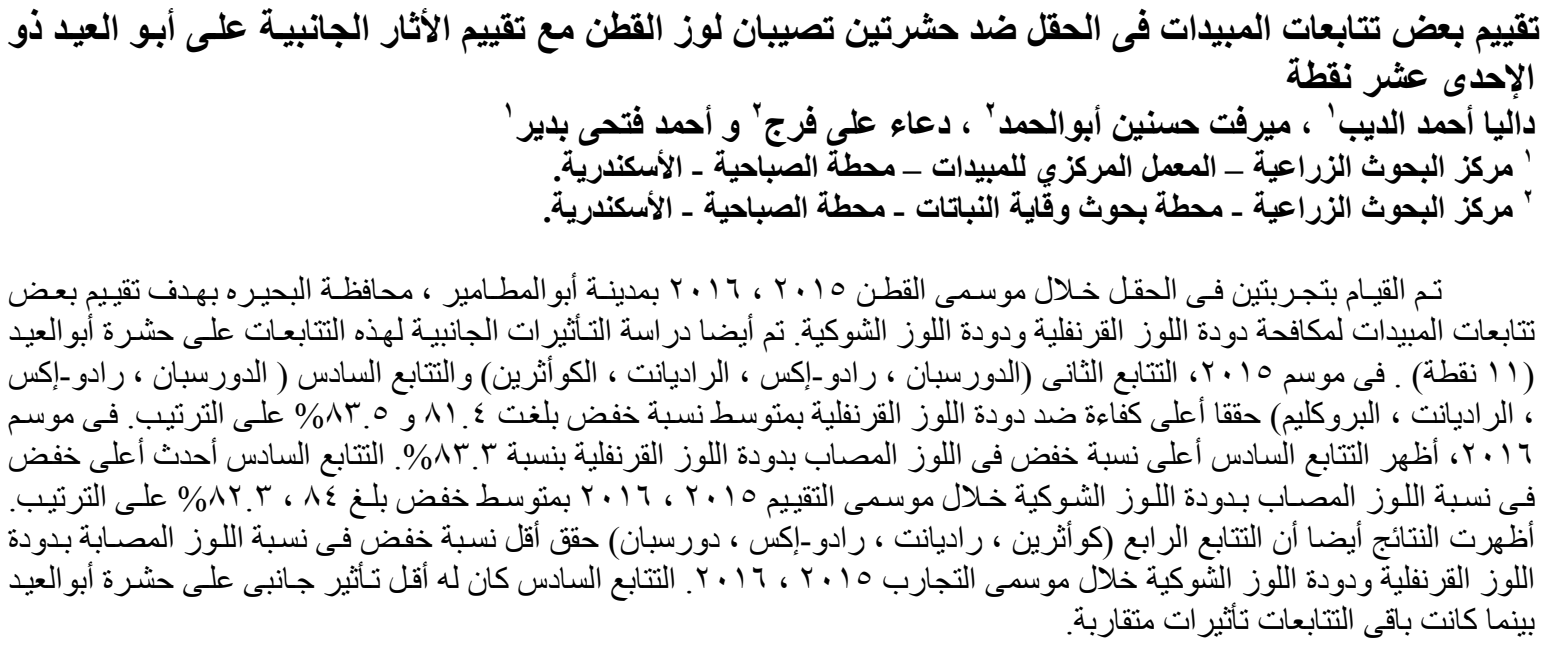

